\section{Impact of idelalisib on health-related quality of life in patients with relapsed chronic lymphocytic leukemia in a phase III randomized trial}

Patients with chronic lymphocytic leukemia (CLL) have overtly impaired well-being relative to healthy controls. ${ }^{1,2}$ Factors associated with lower overall health-related quality of life (HRQoL) in patients with CLL include older age, greater fatigue, severity of comorbid health conditions, advanced disease stage, and ongoing treatment for CLL. ${ }^{2,3}$ The use of standardized patient-reported outcomes has become an increasingly important component of therapeutic assessment in clinical trials, allowing for determination of the impact of treatment on HRQoL. ${ }^{4}$

Idelalisib, a potent, highly selective, oral small-molecule inhibitor of phosphoinositol-3 kinase $\delta$, is approved by the US Food and Drug Administration and the European Medicines Agency, in combination with rituximab, for the treatment of relapsed CLL. ${ }^{5,6}$ In the pivotal randomized, double-blind, placebo-controlled trial of 220 elderly patients with relapsed or refractory CLL and comorbid conditions, idelalisib plus rituximab demonstrated statistically significant and clinically meaningful improvements in overall response rate, progression-free survival, and overall survival, with an acceptable toxicity profile compared with the placebo plus rituximab control. ${ }^{7}$ Grade $\geq 3$ diarrhea, rash, and hepatic transaminase elevations were more frequent in the idelalisib plus rituximab arm.

A prespecified analysis evaluated the impact of idelalis$\mathrm{ib}$ plus rituximab versus rituximab plus placebo on HRQoL - in the absence of the typical chemotherapyrelated toxicity - using the 44-item Functional Assessment of Cancer Therapy-Leukemia (FACT-Leu) scale. The FACT-Leu instrument has well-established psychometric properties $^{8-11}$ that yield a total score and subscales for physical well-being (PWB), functional wellbeing (FWB), social/family well-being (SWB), and emotional well-being (EWB), ${ }^{9}$ and a diagnosis-specific measure for patients with leukemia-specific symptoms (LeuS) such as fevers, chills, night sweats, nodal swelling and fatigue. ${ }^{11}$ The FACT-Leu total score is the sum of all subscales, and the trial outcome index is the sum of the scores for PWB, FWB, and LeuS. Higher scores reflect better HRQoL. The number of items, scoring ranges, and minimally important differences (MID) for each subscale are listed in Online Supplementary Table S1. The survey was administered every 2 weeks until week 8 , every 4 weeks until week 24, every 6 weeks until week 48, and every 12 weeks thereafter until unblinding on November 8,2013 . No HRQoL or performance status data were collected after CLL disease progression. To avoid biasing HRQoL results, the FACT-Leu was administered in person at each visit before other procedures were performed, and before any study information was conveyed to the patient. FACT-Leu was scored based on the Functional Assessment of Chronic Illness Therapy-3 scoring guideline and user manual. ${ }^{12}$

Questionnaire compliance was defined as the proportion of patients who answered at least one question at a scheduled time point relative to all patients available at that time point (i.e., not including patients excluded for other reasons). Missing items in a subscale were imputed. Data collected from the FACT-Leu instrument were not reconciled with adverse event or laboratory data. Repeated measures mixed-effects models were used to assess mean change from baseline within and between treatment arms.

Since a significant portion of the patients had progression of CLL during the study, and no HRQoL or performance status data were collected after disease progression, the duration of data collection varied. Missing data were not imputed for patients lost to follow-up without progression. The varied durations of FACT-Leu score collection were handled by the repeated measure mixed-effects model, which provides robust estimates by analyzing the observed data when data missing at random were reasonably assumed. Kaplan-Meier methods and log-rank tests were applied for each of the FACT-Leu subscale scores, assessing time to changes for each variable. The trial was registered at: clinicaltrials.gov NCT01539512; EudraCT 2011-005180-24.

Between May 2012 and August 2013, 220 patients were enrolled. The study was stopped due to superior efficacy of idelalisib plus rituximab over placebo plus rituximab. Upon study termination and unblinding,

Table 1. Mean differences in patient-reported Functional Assessment of Cancer Therapy-Leukemia outcome score changes from baseline between treatment arms.

\begin{tabular}{|c|c|c|c|c|c|}
\hline \multirow[b]{2}{*}{ Week } & \multicolumn{5}{|c|}{ Mean change from baseline score difference (SEM) } \\
\hline & LeuS & PWB & FWB & TOI & FACT-Leu total \\
\hline 2 & $0.4(1.31)$ & $-0.1(0.65)$ & $0.6(0.80)$ & $1.3(2.38)$ & $1.1(2.96)$ \\
\hline 4 & $2.5(1.33)$ & $0.8(0.66)$ & $1.0(0.81)$ & $4.0(2.41)$ & $4.0(3.01)$ \\
\hline 6 & $2.2(1.37)$ & $0.1(0.68)$ & $1.0(0.84)$ & $2.9(2.48)$ & $3.9(3.09)$ \\
\hline 8 & $3.5(1.43)^{*}$ & $0.6(0.71)$ & $0.7(0.87)$ & $4.6(2.57)$ & $5.2(3.2)$ \\
\hline 12 & $4.7(1.51)^{\dagger}$ & $1.1(0.75)$ & $1.5(0.92)$ & $7.0(2.72)^{\dagger}$ & $6.5(3.39)$ \\
\hline 16 & $5.3(1.66)^{\dagger}$ & $1.9(0.83)^{*}$ & $1.3(1.01)$ & $8.4(2.99)^{\dagger}$ & $9.2(3.72)^{\dagger}$ \\
\hline 20 & $5.4(1.85)^{\dagger}$ & $1.6(0.91)$ & $1.4(1.13)$ & $9.0(3.33)^{\dagger}$ & $9.0(4.14)^{\dagger}$ \\
\hline 24 & $5.0(2.06)^{\dagger}$ & $1.8(1.02)$ & $1.9(1.26)$ & $9.1(3.69)^{\dagger}$ & $10.0(4.58)^{\dagger}$ \\
\hline 30 & $3.0(2.32)$ & $2.1(1.14)$ & $2.6(1.41)$ & $7.7(4.13)$ & $9.6(5.13)$ \\
\hline 36 & $5.1(2.54)^{\dagger}$ & $1.5(1.26)$ & $2.8(1.56)$ & $8.2(4.59)$ & $9.1(5.69)$ \\
\hline 42 & $3.9(3.16)$ & $2.1(1.57)$ & $2.8(1.93)$ & $8.1(5.57)$ & $9.1(6.92)$ \\
\hline 48 & $5.5(3.60)$ & $3.6(1.79)^{\dagger}$ & $3.6(2.20)$ & $12.4(6.32)^{\dagger}$ & $13.1(7.85)$ \\
\hline
\end{tabular}

${ }^{*} P<0.05$ and ${ }^{P} P<0.05$ and exceeded established minimally important difference change scores of 4 (LeuS), 2 (PWB), 5 (TOI), and 6 (FACT-Leu Total) between arms. SEM standard error of the mean; LeuS: leukemia subscale; PWB: physical well-being; FWB: functional well-being; TOI; trial outcome index; FACT-Leu: Functional Assessment of Cancer Therapy-Leukemia. 
A

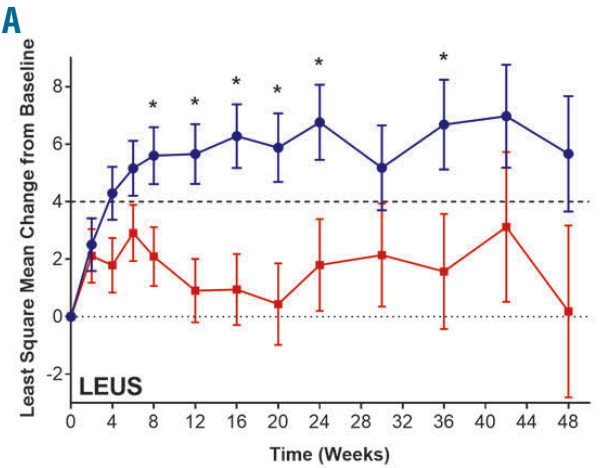

C

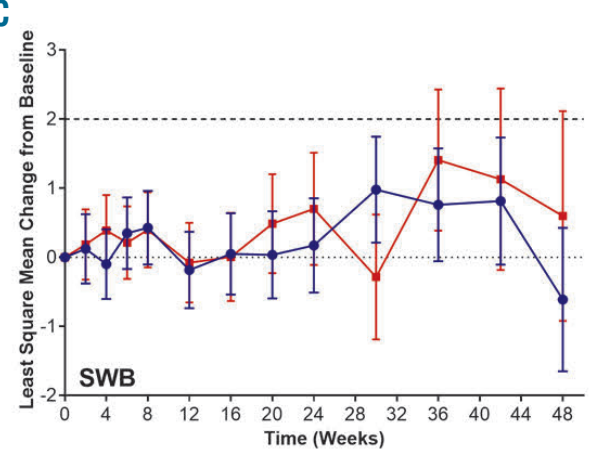

$E$

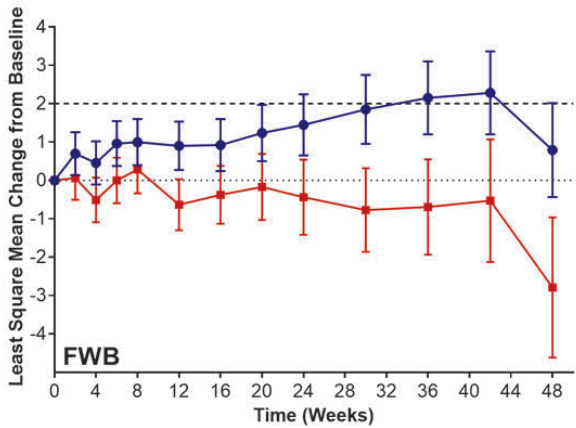

B

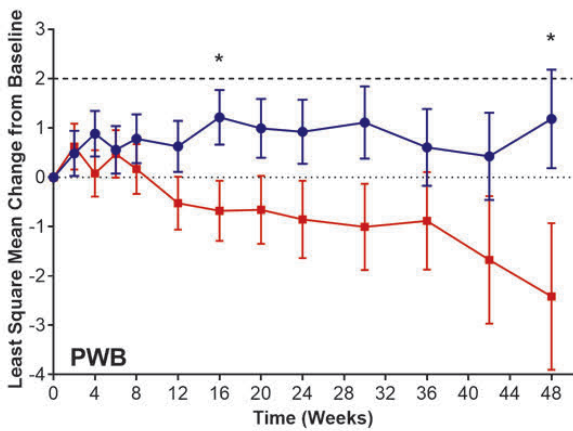

D

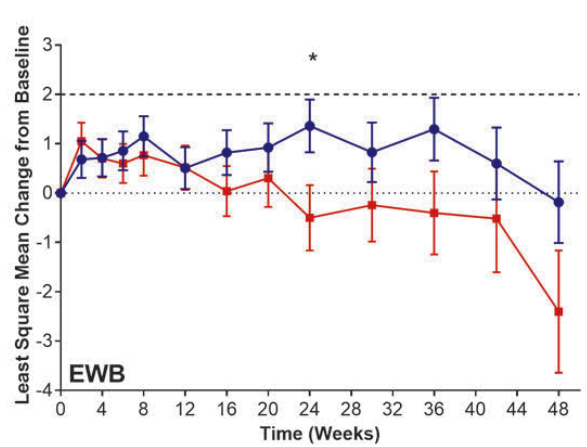

Figure 1. Change in Functional Assessment of Cancer Therapy-Leukemia subscale scores over time between treatment groups. The changes in the mean and standard error of mean scores are shown for: (A) leukemia subscale (LEUS); (B) physical well-being (PWB); (C) social/family well-being (SWB); (D) emotional well-being (EWB); and (E) functional well-being (FWB). Dashed lines correspond to the lower end of the range of minimally important difference. $* P<0.05$ for treatment difference based on a mixed model analysis.

patients could transition to the extension study (NCT01539291) to receive open-label idelalisib monotherapy. The HRQoL data were collected during the blinded phase of the study and analyzed as of the unblinding date of November 8, 2013.

The patients' demographic characteristics were well balanced between the two treatment arms, and betweengroup HRQoL scores were comparable at baseline. The patients' median age was 71 years (range, 47-92) with $78 \%$ of the patients being $\geq 65$ years of age. Forty percent had at least moderate renal dysfunction (creatinine clearance $<60 \mathrm{~mL} / \mathrm{min}$ ), $35 \%$ had cytopenias (grade $\geq 3$ anemia, thrombocytopenia, or neutropenia), and $85 \%$ had a Cumulative Illness Rating Scale (CIRS) score $>6$. The median CIRS score in each arm was 8. Almost two-thirds of patients had advanced-stage disease, and the median time since the initial diagnosis of CLL was approximately 9 years. More than $80 \%$ had unmutated IGHV, and more than $40 \%$ had either del(17p) or TP53 mutations. Patients in both arms had received a median of three prior therapies, including regimens containing rituximab, cyclophosphamide, fludarabine, and bendamustine.

Compliance and subscale completion rates for the FACT-Leu questionnaire across all time points were high and comparable between treatment arms (Online Supplementary Table S2). At week 2, compliance rates in the placebo and idelalisib arms were $93.5 \%$ and $95.5 \%$, respectively, and declined to $70.0 \%$ and $80.8 \%$, respectively, of participants still on treatment by week 48 . Missing data were randomly and evenly distributed in the two arms.

Treatment with idelalisib plus rituximab translated into subjective benefits in patients' HRQoL, particularly for the FACT-Leu PWB and FWB scores, as well as those related to leukemia symptoms. Compared with placebo plus rituximab, treatment with idelalisib plus rituximab led to a significant improvement in the LeuS scores observed already after 8 weeks, and the score difference from baseline exceeded the MID of 4 by 12 weeks $(P<0.05)$. This improvement was maintained in the mixed model through weeks $8,16,20,24$, and 36 in patients available for each assessment (Table 1, Figure 
A

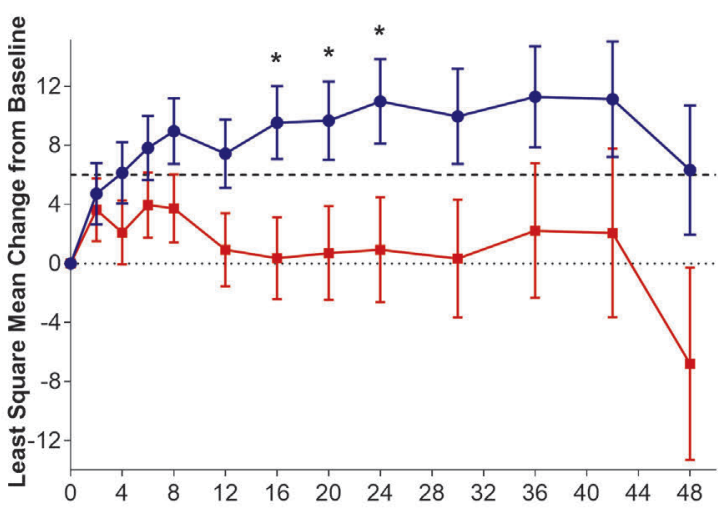

B

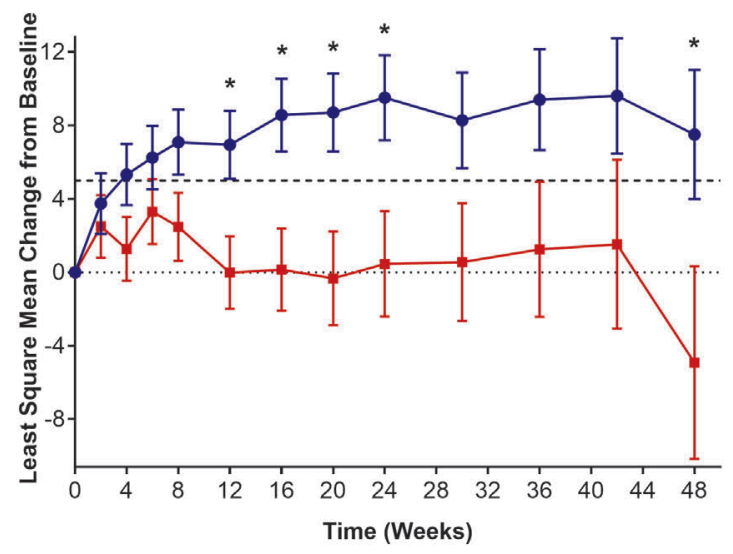

Figure 2. Change in composite measures over time between treatment groups. The change in the mean and standard error of mean scores are shown for: (A) Functional Assessment of Cancer Therapy-Leukemia total score; and (B) trial outcome index. Dashed lines correspond to the lower end of the range of minimally important difference. ${ }^{*} P<0.05$ for treatment difference based on a mixed model analysis.

1A). The overall treatment effect was statistically significant $(P=0.001)$ based on a longitudinal analysis. In the idelalisib plus rituximab group, significant treatment effects were also seen on the PWB $(P=0.015)$ and FWB $(P=0.014)$ subscales (Table 1, Figure $1 \mathrm{~B}, \mathrm{E})$.

EWB and SWB scores did not show any meaningful trend between the two arms $(P=0.08$ and $P=0.77$, respectively) (Figure 1C, D). This may be due to the patients' awareness of potential for rescue in the extension study, or because the psychological burden of having a disease or not achieving a response - was less relevant for patients in this trial, who had a long history of CLL including past treatment failure. Alternatively, patients may have had enough time to learn how to live with a disease that can repeatedly progress and relapse and reached an overall emotional and social plateau that was no longer affected by the treatment.

On the composite scales, patients treated with idelalis$\mathrm{ib}$ plus rituximab scored their HRQoL significantly higher than patients in the placebo plus rituximab group (Figure $2)$. For the trial outcome index (LeuS + PWB + FWB), mixed model analysis showed improvements at weeks
$12,16,20,24$, and $48(P<0.05)$ over the control group, producing a statistically significant and clinically meaningful effect of idelalisib versus placebo on the composite trial outcome index over the entire course of treatment $(P=0.002)$ (Table 1, Figure 2A). A similar improvement was observed in the FACT-Leu total score (LeuS + PWB + $\mathrm{S} / \mathrm{FWB}+\mathrm{EWB}+\mathrm{FWB})$ : patients treated with idelalisib plus rituximab reported significantly higher scores on weeks 16, 20, and $24(P<0.05)$, compared with patients treated with placebo plus rituximab, for a statistically significant and clinically meaningful treatment effect over the entire study $(P=0.006)$ (Table 1 , Figure $2 \mathrm{~B})$. Similarly, time to symptom deterioration was longer in the treatment arm relative to the placebo arm (Online Supplementary Table S3). Improvement in composite scores in the idelalisib plus rituximab arm compared with the placebo plus rituximab arm was seen in both younger ( $<65$ years) and older ( $\geq 65$ years) patients (Online Supplementary Table S4, Online Supplementary Figure S1).

In summary, patients treated with the combination of idelalisib and rituximab had a better HRQoL, possibly associated with response of disease-related symptoms to treatment, beginning as early as 4 weeks after initiation of treatment. This contrasts with HRQoL changes following classic chemoimmunotherapy, when increased HRQoL due to symptomatic improvement from treatment efficacy might be offset in the short term by initial chemotherapy-related toxicity. For example, COMPLEMENT 1 (ofatumumab plus chlorambucil versus chlorambucil alone) and COMPLEMENT 2 (ofatumumab plus fludarabine and cyclophosphamide versus fludarabine and cyclophosphamide)-randomized studies that used chemotherapy as part of the experimental arm showed small and delayed improvements in disease symptoms and HRQoL. ${ }^{13,14}$ In contrast, patients taking idelalisib plus rituximab experienced the HRQoL benefits of disease control without the initial adverse effects of cytotoxicity. Potential limitations include the small numbers of patients available for assessment at later time points (Online Supplementary Table S2), although this was accounted for in the mixed-effects model analysis as the missing data were randomly and evenly distributed between the treatment arms.

Idelalisib plus rituximab is among the first non-cytotoxic treatment regimens for the management of relapsed or refractory CLL. This analysis of patient-related outcomes demonstrates that idelalisib plus rituximab improves not only clinical outcomes but also HRQoL, including a reduction in leukemia-related symptoms and improvements in physical and functional well-being. These data may help patients and clinicians make more informed treatment decisions.

Paolo Ghia, ${ }^{1}$ Steven E. Coutre, ${ }^{2}$ Bruce D. Cheson, ${ }^{3}$ Jacqueline C. Barrientos, ${ }^{4}$ Peter Hillmen, ${ }^{5}$ Andrew R. Pettitt, ${ }^{6}$ Andrew D. Zelenetz, Sanatan Shreay, Michael Hallek ${ }^{9}$ and Richard R. Furman ${ }^{10}$

${ }^{1}$ Università Vita-Salute San Raffaele and IRCCS Ospedale San Raffaele, Milano, Italy; ${ }^{2}$ Stanford School of Medicine, Stanford, CA, USA; ${ }^{3}$ Georgetown University Hospital, Washington, DC, USA; ${ }^{4}$ Zucker School of Medicine at Hofstra/Northwell, Hempstead, NY, USA; ${ }^{5}$ St. James's University Hospital, Leeds, UK; ${ }^{6}$ University of Liverpool, Liverpool, UK; ${ }^{7}$ Memorial Sloan Kettering Cancer Center, New York, NY, USA; ${ }^{8}$ Gilead Sciences, Inc, Foster City, CA, USA; ${ }^{2}$ Department I of Internal Medicine, University Hospital of Cologne, Cologne, Germany and ${ }^{10}$ Weill Cornell Medical College, New York, NY, USA.

Acknowledgments: the authors and Gilead Sciences, Inc., are 
grateful to the late Professor Bertrand Coiffier for his contributions to the study and this manuscript. We thank the patients for their dedication to this clinical trial and the clinical personnel at each of the study sites for their diligence in caring for patients and collecting study data.

Funding: this study was sponsored by Gilead Sciences, Inc. (Foster City, CA, USA). The authors would like to thank Ronald Dubowy, Lyndah Dreiling and Yeonhee Kim, former employees of Gilead Sciences, Inc., for their invaluable assistance with the draft of early stages of this manuscript. Medical writing and editorial assistance was provided by Timothy DiChiara, PhD, of Gilead Sciences, Inc.; and by Ewa Wandzioch, PhD, and Meryl Gersh, PhD, of AlphaBioCom, LLC, King of Prussia (PA, USA), which was funded by Gilead Sciences, Inc.

Correspondence:

PAOLOGHIA - ghia.paolo@hsr.it

doi:10.3324/haematol.2019.238808

\section{References}

1. Else M, Smith AG, Cocks K, et al. Patients' experience of chronic lymphocytic leukaemia: baseline health-related quality of life results from the LRF CLL4 trial. Br J Haematol. 2008;143(5):690-697.

2. Shanafelt TD, Bowen D, Venkat C, et al. Quality of life in chronic lymphocytic leukemia: an international survey of 1482 patients. Br J Haematol. 2007;139(2):255-264.

3. Levin TT, Li Y, Riskind J, Rai K. Depression, anxiety and quality of life in a chronic lymphocytic leukemia cohort. Gen Hosp Psychiatry. 2007;29(3):251-256

4. Efficace F, Gaidano G, Lo-Coco F. Patient-reported outcomes in hematology: is it time to focus more on them in clinical trials and hematology practice? Blood. 2017;130(7):859-866.

5. ZYDELIG® (idelalisib) tablets, for oral use. Full prescribing information. Gilead Sciences, Inc., Foster City, CA, USA. 2018.
6. Zydelig (idelalisib). Summary of product characteristics. Gilead Sciences Ireland UC, Carrigtohill, County Cork, Ireland. 2019.

7. Furman RR, Sharman JP, Coutre SE, et al. Idelalisib and rituximab in relapsed chronic lymphocytic leukemia. N Engl J Med. 2014; 370(11): 997-1007.

8. Cella D, Webster K, Cashy J, et al. Development of a measure of health-related quality of life for non-Hodgkin's lymphoma clinical research: the Functional Assessment of Cancer Therapy - Lymphoma (FACT-Lym). Blood. 2005;106(suppl1):750.

9. Cella DF, Tulsky DS, Gray G, et al. The Functional Assessment of Cancer Therapy scale: development and validation of the general measure. J Clin Oncol. 1993;11(3):570-579.

10. Victorson D, Barocas J, Song J, Cella D. Reliability across studies from the Functional Assessment of Cancer Therapy-General (FACT-G) and its subscales: a reliability generalization. Qual Life Res. 2008; 17(9):1137-1146.

11. Webster K, Chivington K, Shonk C, Eremenco S, Yount S, Hahn E. Measuring quality of life (QOL) among patients with leukemia: the Functional Assessment of Cancer Therapy- Leukemia (FACT-LEU). Qual Life Res. 2002;11(7):678.

12. FACIT.org, 2015. http://www.facit.org/FACITOrg/Questionnaires. Accessed March 09, 2017.

13. Hillmen P, Janssens A, Babu KG, et al. Health-related quality of life and patient-reported outcomes of ofatumumab plus chlorambucil versus chlorambucil monotherapy in the COMPLEMENT 1 trial of patients with previously untreated CLL. Acta Oncol. 2016;55(910):1115-1120.

14. Robak T, Warzocha K, Govind Babu K, et al. Health-related quality of life and patient-reported outcomes of ofatumumab plus fludarabine and cyclophosphamide versus fludarabine and cyclophosphamide in the COMPLEMENT 2 trial of patients with relapsed CLL. Leuk Lymphoma. 2017;58(7):1598-1606. 